\title{
THE DISTRIBUTION OF BCDGS IN VOIDS
}

\author{
ULRICH LINDNER AND KLAUS J. FRICKE \\ Universitätssternwarte, Geismarlandstr. 11, D-37083 Göttingen, Germany \\ AND \\ JAAN EINASTO AND MARET EINASTO \\ Tartu Astrophysical Observatory, EE-2444 Tõravere, Estonia
}

\section{Introduction}

Generally the density of the environment is important for the formation and evolution of galaxies: bright elliptical galaxies reside in the centers of galaxy clusters, whereas late type galaxies preferentially populate small groups, filaments or the outskirts of rich clusters of galaxies. Furthermore, fainter galaxies define smaller voids than bright galaxies. Our investigation of void catalogues in the Northern Local Void region shows that large voids are interlaced by systems of faint galaxies dividing them into smaller subvoids (Lindner et al., 1995). This void hierarchy is an important property of the Large Scale Structure in the nearby Universe and can help to solve problems concerning the distribution of dwarf galaxies and BCDGs.

The questions whether dwarf galaxies are homogeneously distributed in voids or if their distribution reveals any structure is crucial for some theories of galaxy formation, e.g. the biased galaxy formation scenario (Dekel \& Silk, 1986). Moreover, an investigation of the environment of BCDGs may clarify the cause of starbursts and address the question whether BCDGs may constitute a special population of galaxies in voids.

\section{Results}

We investigated the radial distribution of dwarf galaxies and BCDGs inside spherical voids from our void catalogues and performed nearest neighbor tests to study possible differences in the distribution of BCDGs and "normal" dwarf galaxies with respect to bright galaxies.

\subsection{THE RADIAL DISTRIBUTION OF GALAXIES IN VOIDS}

Figure 1 reveals that most faint galaxies are concentrated near void walls. This result has also been found using other methods (Kuhn et al., 1997). The radial distributions are almost identical for all three void catalogues suggesting that the void hierarchy continues to fainter than $M=-18.8$ galaxies (Lindner et al., 1996). The radial distribution of real galaxies in voids differs significantly (99\% confidence level) from random samples which rules out biased galaxy formation scenarios (Dekel \& Silk, 1986).

\subsection{NEAREST NEIGHBOR ANALYSIS}

In Figure 2 we studied unbinned cumulative distributions of nearest neighbor distances for different kinds of galaxies (BCDGs, all galaxies, galaxies brighter than -18.8 and random samples). KS tests confirm that BCDGs are nearer to faint galaxies than to bright galaxies. The nearest neighbor distance distributions for BCDGs and all galaxies are similar (KS: $\approx 76 \%$ ) and differ significantly (99.9\% confidence level) from random samples. The median nearest neighbor distance for BCDGs is about $0.7 \mathrm{~h}^{-1} \mathrm{Mpc}$, which corresponds to a characteristic scale for loose groups or filaments of galaxies. 


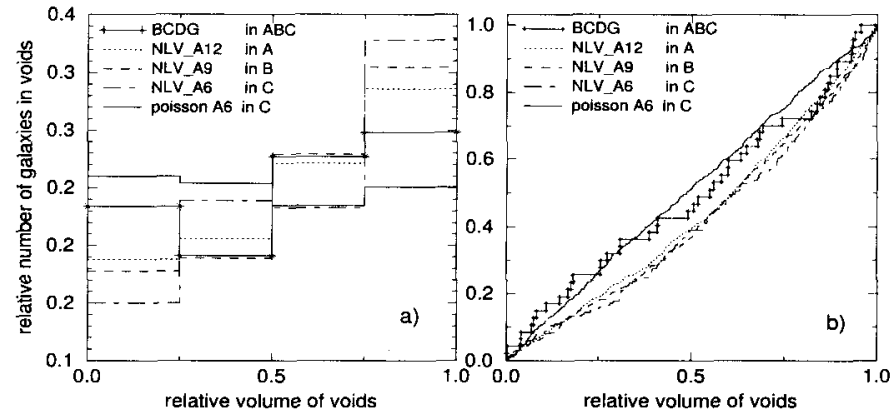

Figure 1. a) differential and b) unbinned cumulative radial distribution of galaxies in voids

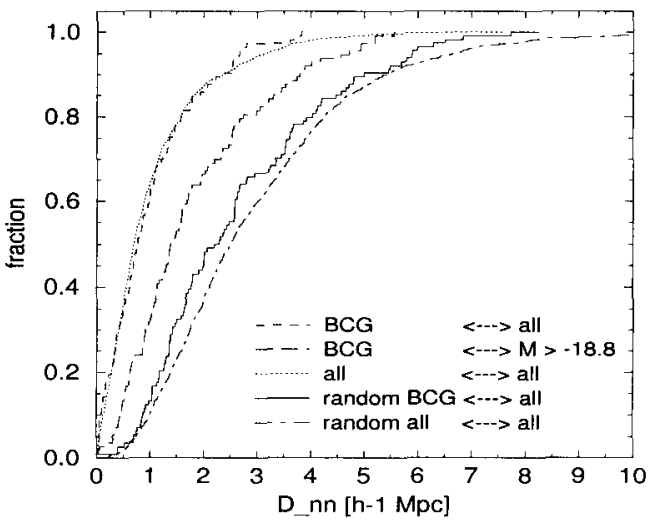

Figure 2. unbinned cumulative distribution of nearest neighbor distances D_nn

\section{Conclusion}

It is generally assumed that BCDGs are LSB dwarf galaxies undergoing a phase of enhanced star formation (Krüger et al., 1991). We found that BCDGs reside preferentially in intermediate density regions, i.e. they avoid high density regions and are not very isolated. This suggests that star bursts may be triggered by neighboring galaxies: high density environment implies high SFR and thus all gas is already consumed. No BCDGs are found in low density environment, suggesting that close neighbors may be needed for BCDG activity. The similarity of the distributions indicates that BCDGs are not an exceptional population of isolated galaxies in voids as it has been suggested previously (Pustil'nik et al., 1995).

\section{References}

Dekel, A., \& Silk, J., 1986, ApJ, 303, 39

Krüger, H., Fritze-von Alvensleben, U., Loose, H.-H., \& Fricke, K.J., 1991, A\&A 242, 343

Kuhn, B., Hopp, U., \& Elsässer, H., 1997, A\&A 318, 405

Lindner, U., Einasto, J., Einasto, M., Freudling, W., Fricke, K.J., \& Tago, E., 1995a, A\& A 301, 329

Lindner, U., Einasto, M., Einasto, J., Freudling, W., Lipovetsky, V.A., \& Richter, G., 1995b, A\&A 314, 1

Pustil'nik, S.A., Ugryomov A.V., Lipovetsky, V.A., Thuan, T.X., \& Guseva, N.G., 1995, ApJ, 443, 449 